\title{
Impacto de la variabilidad climática en la economía de los productores de plátano de comunidades nativas de la Amazonia Peruana
}

\author{
Hugo Guillermo Díaz Panduro \\ dphugocpc@hotmail.com \\ Universidad Nacional de Ucayali \\ Pucallpa - Perú \\ Hugo Díaz Avalos \\ hugodz92@gmail.com \\ Gobierno Regional de Ucayali \\ Pucallpa - Perú \\ Luis José Barboza Hernández \\ bhjose322@gmail.com \\ Gobierno Regional de Ucayali \\ Pucallpa - Perú \\ Sonia María Grecia Méndez García \\ mariagrecia1996@hotmail.com \\ Consultora Civil y Medio Ambiente - CCIMA SAC \\ Pucallpa - Perú
}

\section{RESUMEN}

El objetivo del presente estudio fue analizar la variabilidad climática que genera impacto en la producción y rendimiento del plátano, esto se obtuvo con el análisis de datos meteorológicos de temperatura, precipitación y vientos de la estación meteorológica de la Universidad Nacional de Ucayali y con datos cuantitativos de una encuesta realizada a 66 productores de plátano. El 100\% de los productores encuestados percibió variaciones climáticas, siendo estas, las principales causas de pérdidas en la producción, como es el caso de la comunidad nativa Santa Elisa y el caserío Santa Elena que sufrieron pérdidas económicas entre S/. 310.33 y 1029.37 ocasionadas por el bajo rendimiento de este vegetal. Este bajo y alto rendimiento están relacionados con las variaciones y los eventos climáticos; el incremento de la temperatura hace que el suelo pierda la humedad que las plantaciones necesitan para su adecuado desarrollo, además genera la proliferación de plagas y enfermedades que modifican el proceso de producción. Sin embargo, el alto rendimiento está relacionado con las inundaciones, ya que la creciente de las aguas del río favorece el rendimiento por la cantidad de materia orgánica descompuesta que es acarreada por el caudal fertilizando las orillas donde se cultiva este vegetal.

Palabras clave: Variabilidad climática; impacto económico; elementos meteorológicos. 


\title{
Impact of climate variability on the economy of banana producers from native communities of the Peruvian Amazon
}

\begin{abstract}
The objective of this study was to analyze the climatic variability that generates impact on the production and yield of banana, this was obtained with the analysis of meteorological data of temperature, precipitation and winds from the meteorological station of the National University of Ucayali and with quantitative data from a survey of 66 banana producers. $100 \%$ of the surveyed producers perceived climatic variations, these being the main causes of production losses, as is the case of the Santa Elisa native community and the Santa Elena village, which suffered economic losses between $\mathrm{S} /$. 310.33 and 1029.37 caused by the low yield of this vegetable. This low and high yield are related to variations and climatic events; the increase in temperature causes the soil to lose the humidity that the plantations need for their proper development, and it also generates the proliferation of pests and diseases that modify the production process. However, the high yield is related to the floods, since the rising waters of the river favors the yield due to the amount of decomposed organic matter that is carried by the flow, fertilizing the banks where this vegetable is grown.
\end{abstract}

Keywords: Climate variability; economic impact; meteorological elements.

Artículo recibido: 05 de Mayo 2021 Aceptado para publicación: 20 de Junio 2021 Correspondencia: mariagrecia_1996@hotmail.com Conflictos de Interés: Ninguna que declarar 


\section{INTRODUCCIÓN}

El Perú es uno de los países más vulnerables frente al Cambio Climático. De acuerdo a los indicadores de riesgo climático desarrollados por el Centro Tyndall, se encuentra entre los 10 países con mayor número de personas afectadas desde 1991 al 2000 (Brooks \& Adger, 2003), debido al incremento en los eventos climáticos extremos (El Niño, inundaciones, heladas, huayco, entre otros), la alta dependencia del clima de actividades primarias como la agricultura, así como a una reducida capacidad institucional para ejecutar medidas de adaptación.

Al depender la agricultura de factores climáticos como la temperatura y precipitación, es natural pensar que éstas se verán afectados por las condiciones climáticas, de las cuales cultivos como el plátano son dependientes de las condiciones climáticas y cualquier cambio climático generará impactos en la principal actividad llevando a un riesgo la seguridad alimentaria y la estabilidad económica en regiones donde la agricultura es la base económica.

El plátano es un cultivo de gran importancia económica, es una fuente de trabajo, y una actividad que genera ingresos económicos al agricultor mediante la venta de los vegetales, convirtiéndose en uno de los principales componentes de la canasta familiar de la población amazónica peruana.

El cultivo de plátano afronta una serie de problemas vinculados con factores climáticos (temperatura, precipitación, vientos) factores que inciden en la baja producción y rendimiento, generando una baja rentabilidad principalmente para los productores de plátano del distrito de Masisea. Lo cual esto viene siendo una preocupación para los productores en su zona y es por ello que ha sido fuente de motivación para realizar estudios en los cuales se identifiquen los posibles efectos del cambio climático en la producción del cultivo de plátano y las consecuencias económicas de los posibles cambios en la productividad agrícola.

El objetivo del presente estudio fue Analizar la variabilidad climática que generan impacto en la producción y rendimiento de plátano, determinar el impacto de la variabilidad climática en la producción de plátano e identificar las consecuencias de la variabilidad climática en el rendimiento de plátano en el Distrito de Masisea, Provincia de Coronel Portillo, Región Ucayali. 


\section{MATERIALES Y MÉTODOS}

\subsection{Método de la investigación}

En esta investigación se utilizó el método descriptivo, con la aplicación de este método se describió las causas y consecuencias de la variabilidad climática, así como el impacto de esta en la economía de los productores de plátano.

\subsection{Población y Muestra}

Población: La población de la investigación estuvo conformada por 80 productores de plátano entre los 4 caseríos que pertenecen al Distrito de Masisea las cuales son: Caserío Isla Libertad, Caserío Santa Elena, Caserío Villa el Pescador y Comunidad Nativa Santa Elisa, asimismo, estas 4 zonas productoras generalmente cuentan con grandes extensiones de hectáreas de plátano; tal es así que, estas zonas tienen alguna relación con el impacto de la variabilidad climática en el manejo de sus cultivos y recursos económicos.

Muestra: El tamaño de la muestra se estimó con la siguiente fórmula:

$\mathrm{n}=\frac{\mathrm{N} * \mathrm{Z}^{2} * \mathrm{P} * \mathrm{Q}}{\mathrm{e}^{2}(\mathrm{~N}-1)+\mathrm{Z}^{2} * \mathrm{P} * \mathrm{Q}}$

Donde:

Tamaño de la población: $\mathrm{N}=80$

Valor estandarizado: $\mathrm{Z}=1.96$

Probabilidad de no ocurrencia: $\mathrm{Q}=0.5$

Error muestral: $\mathrm{e}=0.05$

Tamaño de la muestra: $\mathrm{n}=66$

El muestreo se realizó por el método de muestreo aleatorio conglomerado, que consistió en dividir el tamaño de la muestra (66) entre los 4 caseríos que pertenecen al distrito de Masisea, los cuales fueron objetos de la investigación.

Tabla 1. Cantidad de productores de cada caserío que fueron encuestados.

\begin{tabular}{|ll|}
\hline CASERIOS & $\mathrm{N}^{\circ}$ DE ENCUESTADOS \\
\hline CASERÍO ISLA LIBERTAD & 17 productores encuestados \\
\hline COMUNIDAD NATIVA SANTA ELISA & 17 productores encuestados \\
\hline CASERÍO VILLA EL PESCADOR & 16 productores encuestados \\
\hline CASERÍO SANTA ELENA & 16 productores encuestados \\
\hline Total & 66 Productores del distrito de Masisea. \\
\hline
\end{tabular}




\subsection{Procedimiento de recolección de datos}

Para el trabajo en campo nos contactamos con el teniente gobernador de cada caserío para que nos acompañara como guía y de esta manera nos relacione con los productores de plátano; dando a conocer la importancia, el objetivo del trabajo de investigación y posteriormente cuando los productores descansaban de sus faenas aprovechar en aplicar el material de obtención de información.

Para la aplicación de encuestas y entrevistas con los productores de plátano se dependió mucho de los tiempos que ellos tenían para empezar con el trabajo, puesto que se tenía que esperar a que vuelvan de sus chacras para iniciar las actividades. Para empezar con la aplicación de material de recolección de datos primero se les dio a entender los temas respecto a las variabilidades climáticas, sus causas y consecuencias en el sector de la agricultura, luego se direccionaba las preguntas con ejemplos para facilitar el entendimiento relacionado al cambio del clima para así conocer si fueron afectados por las variaciones climáticas a través del tiempo.

En el trabajo en campo para la aplicación del material de recolección de datos se tuvo en cuenta a personas mayores de edad, para obtener información de las variaciones climáticas en una regresión de tiempo con los cambios que ellos han experimentado a través del tiempo.

Es importante señalar que, en el trabajo en campo se presentaron ciertas limitantes metodológicas que generaron cambios en la aplicación de la metodología, la principal fue que el teniente gobernador de cada caserío nos informó que la realización de los talleres solo sería los días sábados o domingos porque de lunes a viernes los productores de plátano acuden a sus chacras a tempranas horas de la mañana y se requiere de su tiempo para que asistan a los talleres, siendo así, no se realizó los talleres y se tuvo que aplicar las encuestas y entrevistas casa por casa.

\subsection{Técnicas e instrumentos para la recolección de datos}

Técnica: La técnica fue la observación estructurada, la cual nos permitió obtener información a través de la encuesta y entrevista, clasificando y determinando los datos recolectados en una situación real.

Instrumentos de recolección de datos: Los instrumentos y materiales que se utilizaron nos permitieron determinar de manera específica y puntual la presencia de datos respecto 
a cada aspecto de la variable de estudio, a continuación, se detalla los equipos y materiales utilizados.

- Encuestas

- Entrevistas

- Estación meteorológica (UNU) para obtener datos meteorológicos de temperatura, precipitación y vientos.

- Libreta de campo

- Lapicero

- Mapas

- Cámara digital

- GPS

\subsection{Procedimiento para la recolección de datos}

\section{Caracterización de la zona de estudio}

El primer día de llegada a los caseríos nos presentamos con el teniente gobernador de cada zona para hacerles saber a qué se debía nuestra presencia en la zona. Posteriormente se dialogó con el teniente gobernador dando a conocer el propósito y la importancia del trabajo de investigación; en tal sentido la coordinación y la visita nos permitió interactuar de manera fluida y a la vez conocer aspectos importantes de la zona de estudio.

\section{Encuestas}

Se aplicaron las encuestas con preguntas semiabiertas para que los productores tengan la libertad en sus respuestas, la estructura de la encuesta estuvo conformada con un total de 28 preguntas distribuidas en dos módulos, la encuesta permitió recoger información sobre los datos generales del encuestado y las variaciones climáticas que se presentan a nivel local. La encuesta sirvió como técnica de obtención de información al personal de la muestra en cada caserío para conseguir respuestas en relación a la variabilidad climática y su impacto en la economía del cultivo de plátano.

\section{Entrevistas}

Las entrevistas se dieron de forma abierta con preguntas simples y concretas, estas fueron estructuradas por el entrevistador basándose en una guía de preguntas específicas para que pueda desarrollarse la conversación con el entrevistado de manera más fluida y de esta manera profundizar aspectos puntuales. La entrevista recopiló información 
concerniente al tercer módulo sobre el tema de la variabilidad climática y sus efectos en la economía.

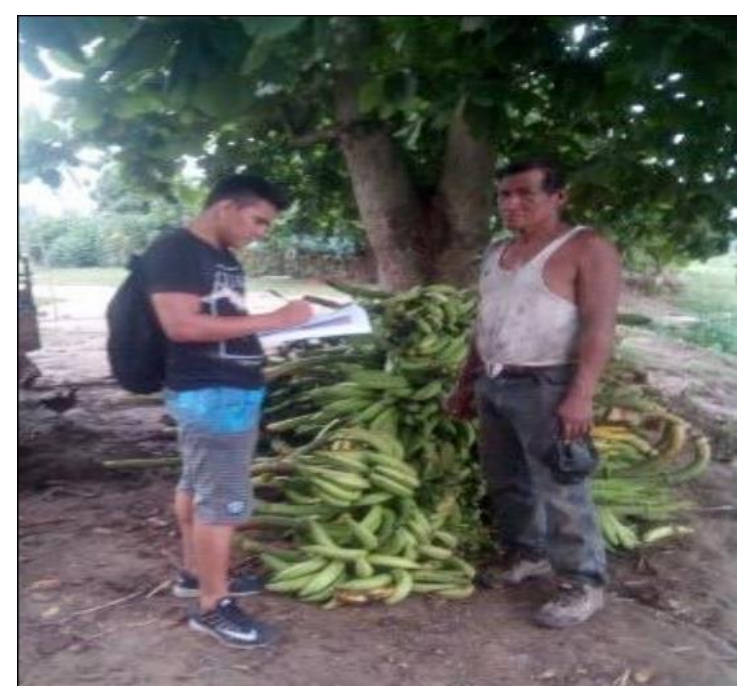

Figura 1. Entrevista a productor de plátano

\section{Obtención de datos meteorológicos}

Se obtuvieron los datos de temperatura, precipitación y vientos de la Estación meteorológica (UNU), siendo una institución pública responsable que participa en la vigilancia atmosférica que nos brindó información en tiempo real y también nos permitió realizar el análisis sobre los elementos meteorológicos que se registran en este órgano.

\subsection{Tratamiento de datos}

Para el tratamiento de datos obtenido de las encuestas y entrevistas se decidió utilizar el Software Microsoft Excel 2016, en este programa se creó un formulario específico para la encuesta desarrollada en campo y posteriormente se procesó la información para obtener las tablas y figuras estadísticas.

\section{RESULTADOS Y DISCUSIÓN}

A partir de las encuestas y entrevistas a los productores de plátano de cuatro caseríos que pertenecen al distrito de Masisea fue posible describir: que del total de los 66 encuestados el $100 \%$ eran varones. En cuanto a la distribución de los encuestados por edades se tiene que el 59\% está representado por personas mayores de 46 años. En lo referente al nivel educativo el $64 \%$ de los encuestados tienen estudios primarios, el 35\% tienen estudios secundarios y el $1 \%$ tiene estudio superior (Tabla 2). Respecto al tipo de vivienda; la mayoría de los productores tienen viviendas estructuralmente estables, ya que el $73 \%$ tiene un tipo de vivienda de madera con techo de calamina, el $15 \%$ es de madera con techo de paja, otros tipos de viviendas se describen en la tabla 2. Respecto al tamaño de 
su fundo el $1 \%$ de los productores tiene entre 150 has, el 5\% tiene entre 20 a 40 has, mientras que el $2 \%$ de los productores tiene entre 10 a 18 has y el $92 \%$ tiene entre media ha a 10 has.

Tabla 2. Datos generales de los productores de plátano en el distrito de Masisea

\begin{tabular}{|c|c|}
\hline \multicolumn{2}{|c|}{ Datos generales del encuestado } \\
\hline 1. Sexo & Masculino (100\%); femenino (0\%) \\
\hline 2. Edad & $\begin{array}{l}\text { Más de } 60 \text { años (12\%); } 46 \text { a } 59(47 \%) ; 36 \\
\text { a } 45(36 \%) ; 30 \text { a } 35(5 \%)\end{array}$ \\
\hline 3. Nivel educativo & $\begin{array}{l}\text { Primaria }(64 \%) ; \text { secundaria }(35 \%) \text {; } \\
\text { superior }(1 \%) \text {. }\end{array}$ \\
\hline 4.Tipo de vivienda & $\begin{array}{l}\text { Madera con techo de calamina }(73 \%) \text {; } \\
\text { madera con techo de paja }(15 \%) \text {; cercado } \\
\text { con manta con techo de calamina }(8 \%) \text {; } \\
\text { cercado con caña brava con techo de } \\
\text { calamina }(2 \%) \text {; seminoble con techo de } \\
\text { calamina }(1 \%) \text {; triplay con esteras con } \\
\text { techo de calamina }(1 \%)\end{array}$ \\
\hline 5. Tamaño de su fundo & $\begin{array}{l}150 \text { has }(1 \%) ; 20 \text { a } 40 \text { has }(5 \%) ; 10 \text { a } 18 \\
\text { has }(2 \%) ; \text { media ha a } 10 \text { ha }(92 \%)\end{array}$ \\
\hline
\end{tabular}

Obtener información de los datos generales nos permitió conocer los indicadores más relevantes para apreciar las características socioeconómicas en la que se encuentran los productores de cada zona, de las cuales tal como se ha mencionado en la tabla 2, la mayoría de los productores tienen viviendas estructuralmente estables, esto refleja que los productores se encuentran en buenas condiciones ya que es un factor que va de la mano con la educación, siendo la educación un componente importante del desarrollo económico, que a través de ello permite también a los productores tener mayor conciencia de los cambios que se presentan en su zona. Asimismo, la importancia de la educación en el bienestar económico siempre ha sido un factor de desarrollo que ha permitido preparar a las personas y de esta manera tener mayores posibilidades en el desempeño de sus trabajos ya que la educación aumenta el conocimiento de muchas técnicas, mejora la capacidad del individuo, promoviendo la división del trabajo y el empleo de maquinaria, y con ello, mejora la productividad del sistema económico y social (UNESCO, 1968). 
Variabilidad climática que genera impacto en la producción y rendimiento de plátano.

Los 66 productores de plátano respondieron un cuestionario sobre la variabilidad climática en su zona con el propósito de conocer el impacto de estas variaciones en su economía, la cual se basa en la producción y rendimiento del cultivo de plátano. Obteniéndose como resultado que el $100 \%$ de los productores de plátano han percibido variaciones climáticas en su distrito. Del total de 66 productores encuestados, el $70 \%$ afirman que hace 5 años se viene presentando esas variaciones climáticas (temperatura, precipitación, vientos), el $29 \%$ y $1 \%$ afirman que han percibido variaciones hace 10 y 20 años, respectivamente, tal es así que estas variaciones han generado impacto en la producción y rendimiento del cultivo de plátano. Esto concuerda con lo señalado por Vergara (2011) que realizó un estudio con comuneros de la comunidad de Conchucos Ancash, sobre variabilidad climática, en el cual, los campesinos al igual que los productores de plátano del distrito de Masisea han percibido variaciones climáticas a lo largo de los años, principalmente en la temperatura y precipitación, afectando sus cultivos (papas nativas), reduciendo así mismo su potencial económico.

\section{Eventos climáticos frecuentes}

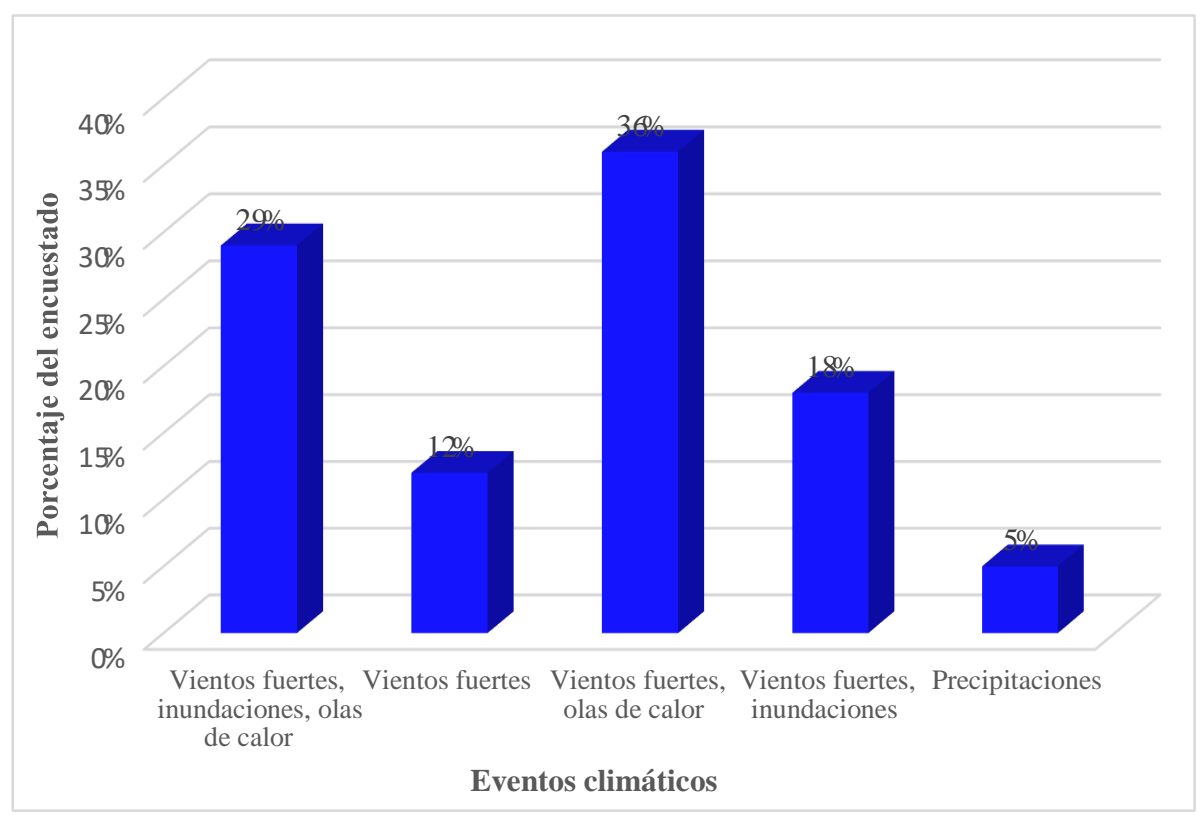

Gráfico 1. Eventos climáticos que se presentan con mayor frecuencia en la zona de los productores de plátano 
En el gráfico 1, se puede observar los eventos climáticos que se presentan con mayor frecuencia. Según los reportes del (Ministerio de Agricultura, datos no publicados) las inundaciones son constantes en época de creciente y esto de evidencia con el promedio máximo de 145.705 msnm que alcanza en el periodo de febrero - abril (2005-2015), esto representa un impacto considerable en la producción y rendimiento del plátano ya que, en los meses de creciente este cultivo se encuentra en el periodo de desarrollo de los dedos de los racimos el cual se ve amenazado por este evento.

A continuación, presentamos la variabilidad de algunos elementos meteorológicos con datos obtenidos de la estación meteorológica de la Universidad Nacional de Ucayali durante 31 años y también el evento climático de las inundaciones el cual es un dato obtenido del Ministerio de Agricultura, a través de ello se realizó el análisis para conocer el impacto en la producción y rendimiento del cultivo de plátano.

\section{Variables climáticas}

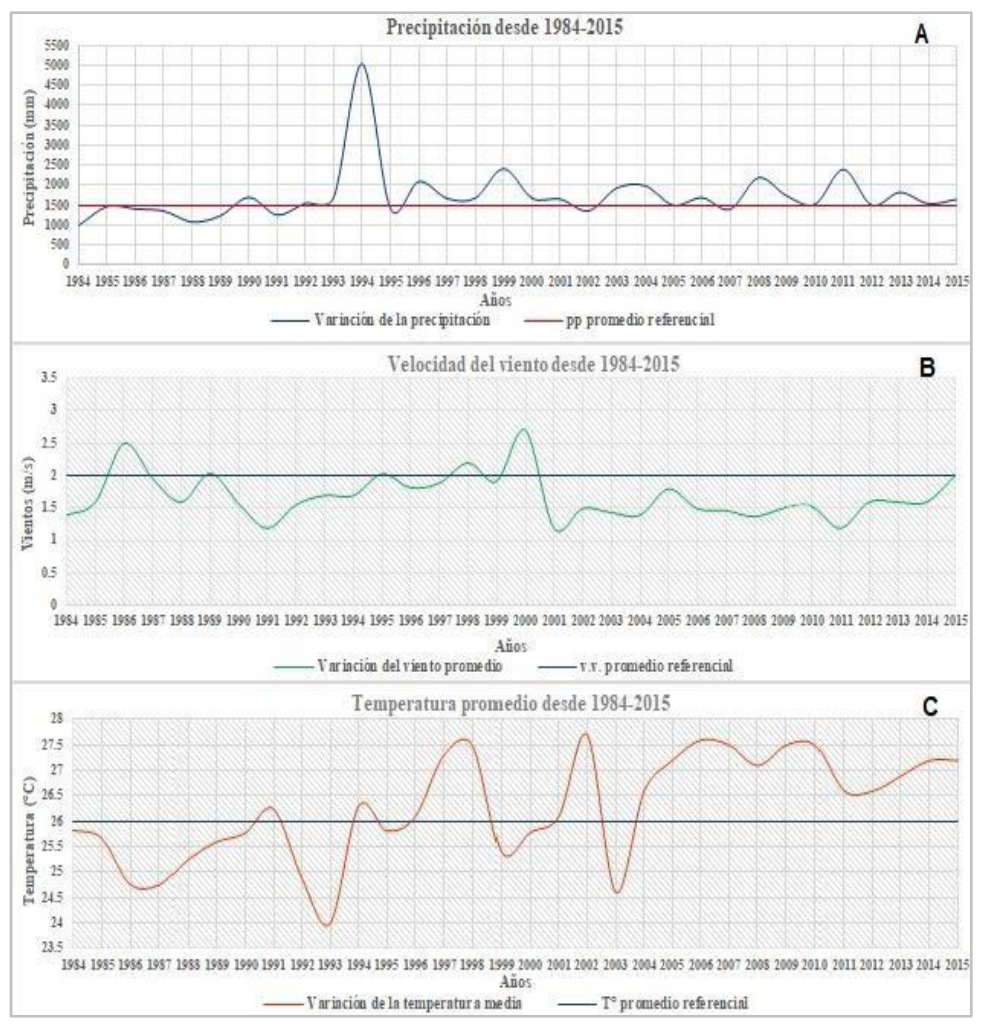

Gráfico 2. Variaciones climáticas de precipitaciones, vientos, temperaturas. Fuente:

(SENAMHI-UNU, 2017) 


\section{Evento climático}

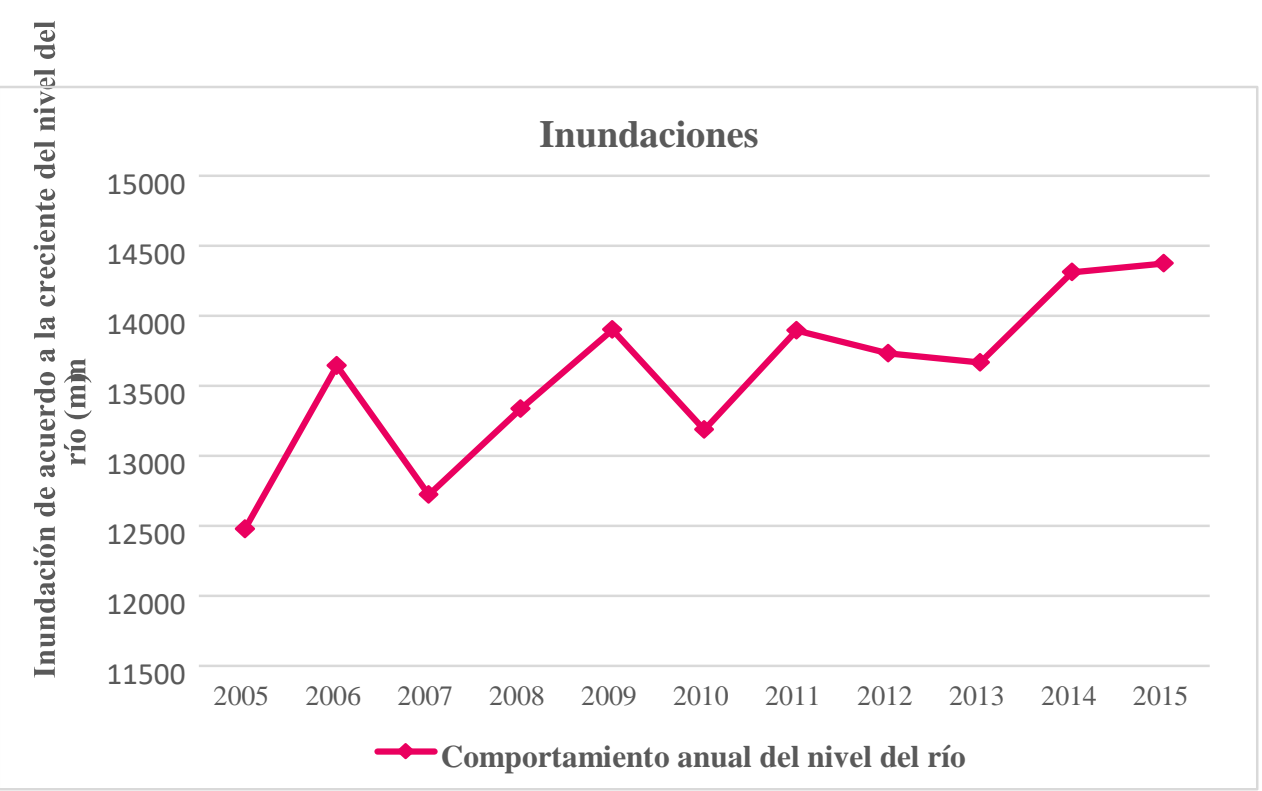

Gráfico 3. Comportamiento de las inundaciones. Fuente (OEIA-DRAU, 2017)

\section{Producción y Rendimiento anual del cultivo de plátano}

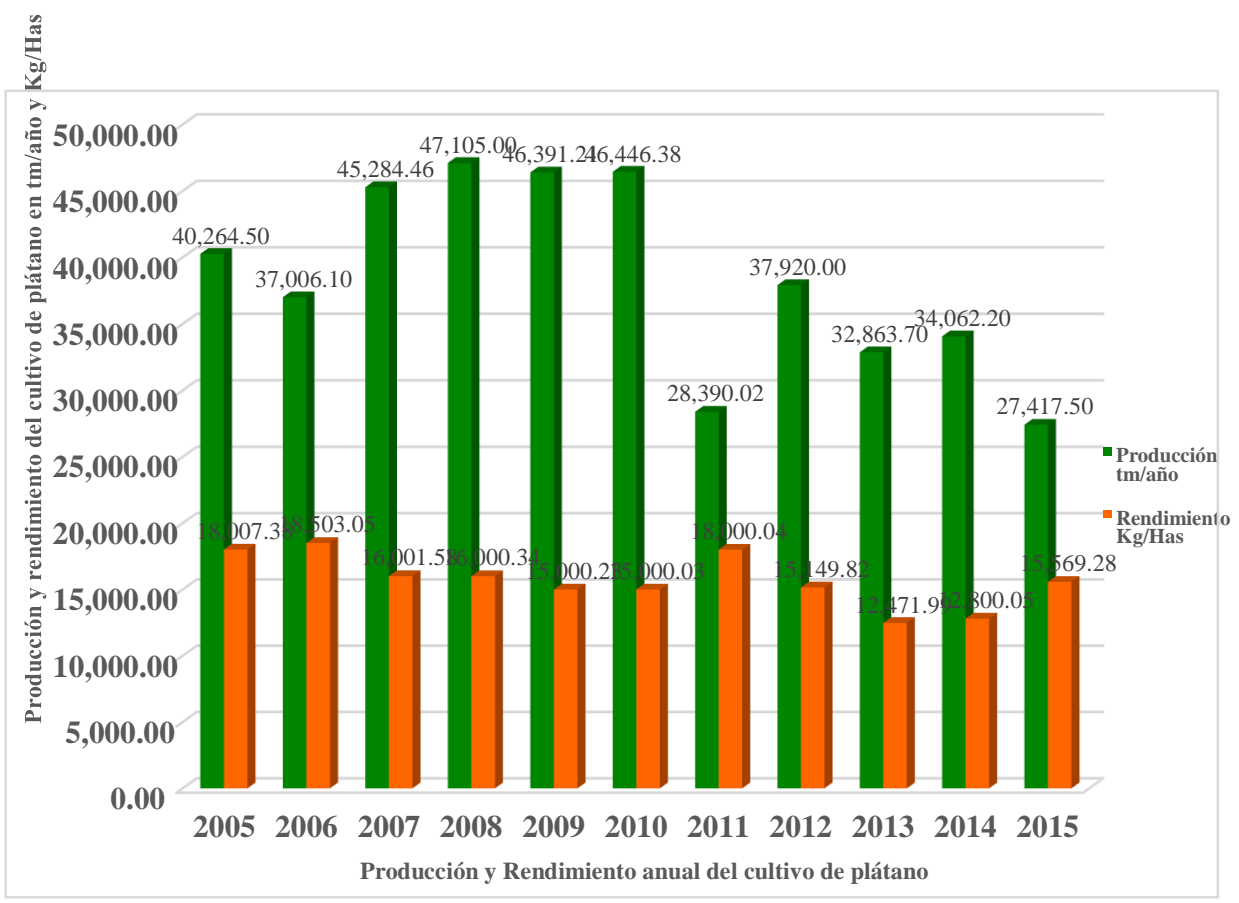

Gráfico 4. Producción y rendimiento anual del cultivo de plátano en el distrito de Masisea. Fuente: (OEIA-DRAU, 2017) 
A partir de estos datos proyectados fue posible describir la influencia de estos eventos climáticos en la producción y rendimiento del cultivo y sus efectos económicos.

En el gráfico 4, se puede observar que en el periodo 2005-2010 hubo una alta producción y rendimientos regulares, pero el año 2011 hubo una baja producción y un alto rendimiento debido a que ese año la región Ucayali fue abordada por una inundación inusual, la cual afectó a los platanales mermando su población por el ahogamiento de los mismos y por la caída de estos; los que se pudieron salvar fueron mejorados por la abundancia de materia orgánica descompuesta favoreciendo así su rendimiento, posteriormente la producción continuó en forma irregular y se tuvo que instalar nuevas plantaciones en forma desigual porque los rendimientos son motivados por el factor clima ya que nuestra agricultura se desarrolla por secano (depende de las lluvias) y de la creciente del río Ucayali ya que sus aguas al transcurrir por su caudal va recogiendo diferente clases de limos, los cuales van fertilizando a nuestra cementera dado que la producción de plátano en la región Ucayali es puramente orgánica, no se utiliza fertilizantes ni abonos para su producción (OEIA-DRAU, 2017).

\section{Impacto de la variabilidad climática en la producción de plátano.}

Es preciso mencionar lo señalado por los productores del distrito de Masisea, que a lo largo del tiempo llevan viviendo en la zona, van cultivando constantemente la chacra y con el paso del tiempo han ido percibiendo las variaciones climáticas cuyos impactos negativos se vieron reflejados en su economía, afirman que a medida que se presentan fuertes vientos, estos terminan tumbando de raíz a los platanales generando pérdidas de plantaciones y en algunos casos llevando casi todos sus cultivos de plátanos, eso genera un impacto en la economía. Asimismo, en época de creciente (febrero-abril) se inundan los platanales llegando a permanecer así más de 15 días lo cual produce el ahogamiento de los platanales, ocasionando pérdidas en las plantaciones y perjudicando la economía del agricultor amazónico. También agregaron que estos últimos años por el incremento de la temperatura se retrasa el desarrollo de los platanales, es decir; no les permite engordar los dedos de los racimos, porque el calor ocasiona que el suelo se seque y quite la humedad que los platanales necesitan para su adecuado desarrollo y eso genera bajo rendimiento de la cosecha por lo tanto también perjudica la economía.

También es de gran importancia señalar que en estos últimos años por el incremento de la temperatura aparecieron muchas plagas y enfermedades como la cigatoca negra que 
seca la hoja lo que impide que los dedos de los racimos engorden, el cornegacho que se introduce por el tallo y termina secando las hojas y eso hace que se tengan que sacar las plantaciones y volver a resembrar nuevos hijuelos, también el gusano conocido como picudo, que es un gusano torneador se come el tallo de la planta. La aparición de estas plagas y enfermedades muestran que el incremento del calor no solo ha ocasionado el empobrecimiento del suelo, sino también la proliferación de vectores siendo un factor limitante en la producción y rendimiento del cultivo de plátano y que en estos últimos años ha generado impactos en la economía de los productores de plátano.

Por esto fue de gran importancia estimar el porcentaje de estas pérdidas; para ello fue necesario conocer la producción de racimos/año de cada productor para así conocer sus ingresos y posteriormente se realizó el cálculo en promedio de cada caserío y también el promedio de sus ingresos, esto nos permitió conocer las pérdidas económicas en porcentaje de cada caserío. El costo unitario por cada racimo de plátano fluctúa según el tamaño y de acuerdo a la época de cosecha (invierno o verano). En época de invierno se incrementa la oferta de este producto, presentándose problemas de sobreproducción llegando a costar un racimo chico desde S/. 2.00 a 3.00 soles y el racimo grande hasta S/. 6.00 soles. Sin embargo, en época de verano este producto llega a escasear por lo que baja la oferta y se eleva el precio desde S/. 5.00 a 7.00 soles el racimo chico y el racimo grande desde S/. 10.00 a 15.00 soles. Asimismo, para la estimación del ingreso de cada productor se consideró el promedio del costo unitario el cual es de S/. 5.00 soles de acuerdo a la información de los productores de plátano, de las cuales se multiplicó la cantidad de racimos/año por el promedio del costo unitario y se estimó el ingreso para cada productor. Asimismo, con la información proporcionada por los productores de plátano, se generó una proyección del porcentaje de pérdidas económicas (soles) en base al porcentaje de pérdidas que han sufrido los productores en su zona ya que esto nos permitió analizar y conocer el impacto de las variaciones climáticas en la economía de los productores de plátano. 
Tabla 3. Proyección de pérdidas en la producción del cultivo de plátano.

\begin{tabular}{|c|c|c|c|}
\hline \multicolumn{5}{|c|}{ CASERÍO } \\
\hline ISLA LIBERTAD & $\begin{array}{c}\text { CC.NN SANTA } \\
\text { ELISA }\end{array}$ & $\begin{array}{c}\text { CASERIO VILLA EL } \\
\text { PESCADOR }\end{array}$ & $\begin{array}{c}\text { CASERIO SANTA } \\
\text { ELENA }\end{array}$ \\
\hline 1552.9412 & 1379.294 & 3162.5 & 3162.5 \\
\hline \multicolumn{4}{|c|}{ S/. 5.00 } \\
\hline 3162.5 & 6896.47 & 15812.5 & 22875 \\
\hline 388.23 & 344.82 & 790.62 & 1143.75 \\
\hline 776.47 & 689.64 & 1581.25 & 2287.5 \\
\hline 1552.94 & 1379.29 & 3162.5 & 4575 \\
\hline 2329.41 & 2068.94 & 4743.75 & 6862.5 \\
\hline 3882.35 & 3448.23 & 7906.25 & 11437.5 \\
\hline 4658.82 & 4137.88 & 9487.5 & 13725 \\
\hline 5435.29 & 4827.52 & 11068.75 & 16012.5 \\
\hline 6211.76 & 5517.17 & 12650 & 18300 \\
\hline 6988.23 & 6206.82 & 14231.25 & 20587.5 \\
\hline
\end{tabular}

Según los productores encuestados al preguntarles qué porcentaje representan sus pérdidas con respecto al cultivo de plátano, respondieron que esas pérdidas se presentan en un $5 \%, 10 \%, 20 \%, 30 \%, 50 \%, 60 \%, 70 \%, 80 \%$ y en algunos casos hasta el $90 \%$ tal como se muestra en la tabla 3 . Por ejemplo, los productores que señalaron sus pérdidas en un 5\% sufrieron pérdidas económicas desde S/. 344.82 a 1143.75 soles que representa a la comunidad nativa Santa Elisa y el caserío Santa Elena. Sin embargo, los productores que manifestaron sus pérdidas en un $90 \%$ se han visto afectados en casi todos sus cultivos ocasionándoles pérdidas económicas hasta S/. 20587.5 soles como es el caso del caserío Santa Elena. Estas pérdidas económicas se deben principalmente a las variaciones climáticas de temperatura, precipitación, vientos y los eventos climáticos como las inundaciones ya que estas condiciones climáticas son las que se han presentado frecuentemente en su zona. El cultivo de plátano es dependiente a las condiciones climáticas cuyas variaciones están relacionados a las actividades que viene desarrollando el agricultor Amazónico en su Distrito, por su gran intensidad tiene efectos ambientales que ha ocasionado alteración en el proceso de producción del cultivo llevando a un riesgo de seguridad alimentaria para el poblador de la selva. 


\section{Pérdidas en el rendimiento de la cosecha de plátano}

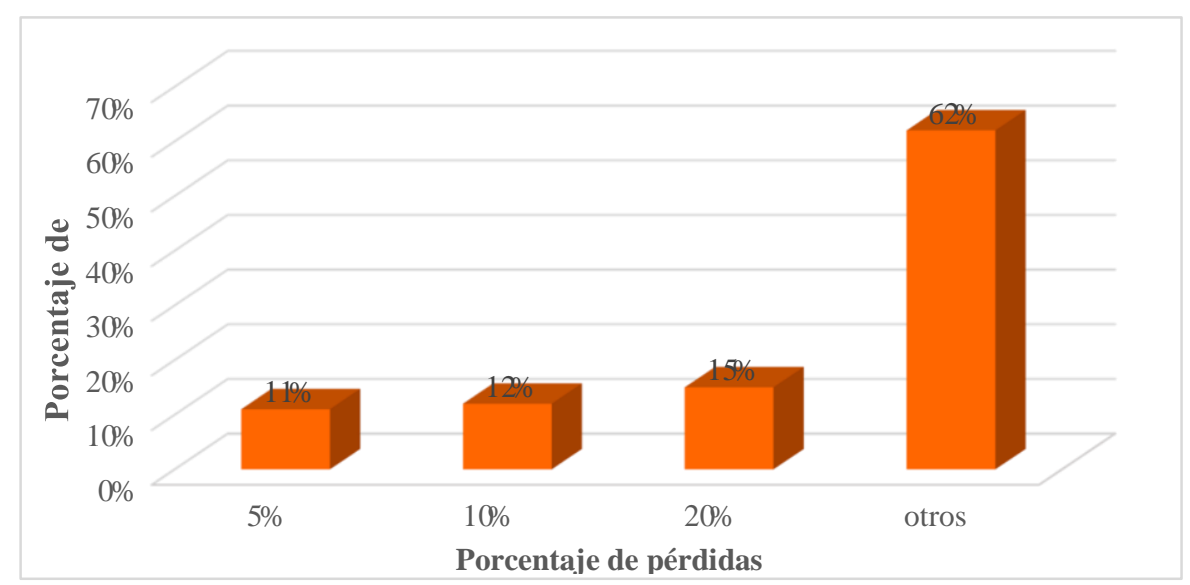

Gráfico 5. Porcentaje de pérdidas respecto al rendimiento de la cosecha de plátano.

En el gráfico 5, se puede observar las pérdidas en el rendimiento de la cosecha de plátano, tal es así que, el 11\% de los productores afirmaron que sus pérdidas se dieron en un 5\% en el rendimiento de la cosecha de plátano, el 12\% de los productores afirman que sus pérdidas se dieron en un $10 \%$, mientras que el $15 \%$ afirman que sus pérdidas se dieron en un $20 \%$ y el $62 \%$ afirman que sus pérdidas se dieron en más de $20 \%$. En tal sentido los productores señalaron que estas pérdidas se deben al incremento de la temperatura, ya que el calor no permite el engorde de los dedos de los racimos y eso ha ocasionado un bajo rendimiento generándoles pérdidas no solo económicas sino también en el empleo, comentaron que en muchas ocasiones por falta de empleo tenían que buscar trabajos temporales para subsistir hasta que se mejore la situación de sus chacras. Pues según (CEPAL, 2013) las actividades agrícolas resultan particularmente sensibles a las condiciones climáticas. Los resultados obtenidos en ciertos productos específicos son bastante heterogéneos y presentan una alta incertidumbre. Por ejemplo, a raíz del cambio climático se modifican los rendimientos del arroz estimados para 2050: oscilan entre una pérdida del $3 \%$ y un aumento del 2\%, según el escenario utilizado. En el caso de otros cultivos, como la yuca, el plátano, la batata (camote) y el tomate, en 2050 se espera que los rendimientos mengüen entre un $1 \%$ y un $30 \%$, también dependiendo del escenario. Según (Vergara \& otros, 2013) estas pérdidas traen aparejadas consecuencias negativas para el empleo en el sector agropecuario y la seguridad alimentaria regional, así como un mayor desbalance en el sector externo y un potencial aumento de los precios, que afectará sobre todo a los pobres. 
A raíz de las variaciones climáticas que generan bajo rendimiento en el cultivo de plátano, se pudo estimar las pérdidas en el rendimiento de este vegetal de acuerdo a los porcentajes de pérdidas que los productores han manifestado en el transcurso de los años que han experimentado esas variaciones. A continuación, se presenta la proyección de pérdidas en el rendimiento por las variaciones climáticas y también por los eventos climáticos identificados en el estudio.

Tabla 4. Proyección de pérdidas en el rendimiento del cultivo de plátano

\begin{tabular}{|c|c|c|c|c|c|}
\hline & \multicolumn{4}{|c|}{ CASERÍO } \\
\hline & & $\begin{array}{l}\text { ISLA } \\
\text { LIBERTAD }\end{array}$ & $\begin{array}{l}\text { CC.NN SANTA } \\
\text { ELISA }\end{array}$ & $\begin{array}{c}\text { CASERIO VILLA } \\
\text { EL PESCADOR }\end{array}$ & $\begin{array}{c}\text { CASERIO } \\
\text { SANTA } \\
\text { ELENA }\end{array}$ \\
\hline \multicolumn{2}{|c|}{$\begin{array}{l}\text { PROMEDIO DEL } \\
\text { PESO DE RACIMOS } \\
\text { (Kg/Has) }\end{array}$} & 23294.12 & 20689 & 47438 & 68625 \\
\hline \multicolumn{2}{|c|}{$\begin{array}{l}\text { PRECIO EN CHACRA } \\
\text { (céntimos) }\end{array}$} & \multicolumn{4}{|c|}{ S/. 0.30} \\
\hline \multicolumn{2}{|c|}{$\begin{array}{c}\text { PROMEDIO } \\
\text { INGRESOS (soles) }\end{array}$} & 6988.23 & 6206.7 & 14231.4 & 20587.5 \\
\hline \multirow{9}{*}{ PÉRDIDAS } & $5 \%$ & 349.41 & 310.33 & 711.57 & 1029.37 \\
\hline & $10 \%$ & 698.82 & 620.67 & 1423.14 & 2058.75 \\
\hline & $20 \%$ & 1397.64 & 1241.34 & 2846.28 & 4117.5 \\
\hline & $30 \%$ & 2096.46 & 1862.01 & 4269.42 & 6176.25 \\
\hline & $50 \%$ & 3494.11 & 3103.35 & 7115.7 & 10293.75 \\
\hline & $60 \%$ & 4192.93 & 3724.02 & 8538.84 & 12352.5 \\
\hline & $70 \%$ & 4891.76 & 4344.69 & 9961.98 & 14411.25 \\
\hline & $80 \%$ & 5590.58 & 4965.36 & 11385.12 & 16470 \\
\hline & $90 \%$ & 6289.40 & 5586.03 & 12808.26 & 18528.7 \\
\hline
\end{tabular}

En la tabla 4, se puede observar la proyección de pérdidas en el rendimiento del cultivo de plátano. Para el cálculo de las pérdidas en el rendimiento de plátano se tuvo en cuenta el promedio de dedos por racimo el cual es de 60 dedos y el peso por cada dedo que es de 250 g según la información proporcionada por los productores de plátano, de las cuales teniendo como base estos datos obtenidos; primero se estimó el peso de los racimos producidos al año por cada productor y luego se sacó el promedio del peso de cada caserío. Seguido con el promedio obtenido se multiplicó con el promedio del peso de racimos (Kg/Has) por el promedio del precio por unidad de plátano que es de $\mathrm{S} / 0.30$ 
céntimos y se obtuvo el promedio de los ingresos para cada caserío. Posteriormente para la estimación de las pérdidas en el rendimiento de plátano se realizó el cálculo del porcentaje de pérdidas en base al promedio de los ingresos multiplicado por el porcentaje de pérdida.

El porcentaje de pérdidas tal como se ha mencionado anteriormente fue de 5\%,10\%, $20 \%, 30 \%, 50 \%, 60 \%, 70 \%, 80 \%$ y $90 \%$ según lo proporcionado por los productores de plátano, en base a estos porcentajes de pérdidas se puede observar las pérdidas en el rendimiento del cultivo de plátano tal como se muestra en la tabla 4. Tanto así que los productores que manifestaron sus pérdidas en un 5\% sufrieron pérdidas económicas por el bajo rendimiento desde S/. 310.33 a 1029.37 soles como es el caso de la comunidad nativa Santa Elisa y el caserío Santa Elena. Los productores que manifestaron sus pérdidas en un $90 \%$ sufrieron pérdidas económicas de hasta S/.18 528.75 soles como es el caso del caserío Santa Elena. El bajo y alto rendimiento están relacionado con las variaciones climáticas y los eventos climáticos, tal es así que, la variable climática como el incremento de la temperatura hace que el suelo pierda la humedad que las plantaciones necesitan para su adecuado desarrollo generando un retraso en el engorde de los dedos de los racimos el cual se traduce en un bajo rendimiento, también genera la proliferación de plagas y enfermedades la cual modifica el proceso de producción del plátano alterando la cosecha. Sin embargo, el alto rendimiento está relacionado con el evento climático de las inundaciones en el cual la creciente de las aguas del río favorece el rendimiento de plátano por la cantidad materia orgánica descompuesta que acarrea el rio al transcurrir por su caudal fertilizando las orillas donde se cultiva este vegetal.

\section{CONCLUSIONES}

- Las variables climáticas que generan impacto en la producción y rendimiento del cultivo de plátano son la temperatura, precipitación y vientos, siendo estas variaciones las que están relacionadas con la economía de los productores de plátano, ya que sus cultivos dependen de las condiciones climáticas y cualquier cambio de estas variaciones generará un impacto significativo en la producción y rendimiento de este vegetal. Las variables climáticas fueron comparadas con los datos meteorológicos de temperatura, precipitación y vientos de la estación meteorológica de la Universidad Nacional de Ucayali. 
- El impacto en la producción del cultivo de plátano es ocasionado por las variaciones climáticas cuyo comportamiento ha generado alteración en el desarrollo de estas plantaciones llevando a un impacto en la economía de los productores de plátano, los impactos identificados son: pérdida de plantaciones, disminución de la producción de plátano y pérdida en la economía. Los productores de plátano manifestaron sus pérdidas en porcentaje estas se dieron en un 5\%, 10\%, 20\%, 30\%, 50\%, 60\%, 70\%, $80 \%$ y $90 \%$. Asimismo, los productores que señalaron sus pérdidas en un 5\% sufrieron pérdidas económicas desde S/. 344.82 a 1143.75 soles como es el caso de la comunidad nativa Santa Elisa y el caserío Santa Elena. Sin embargo, los productores que manifestaron sus pérdidas en un $90 \%$ sufrieron pérdidas económicas de hasta S/. 20587.5 soles como es el caso del caserío Santa Elena.

- Se identificó las consecuencias de la variabilidad climática en el rendimiento del cultivo de plátano el cual fueron estimados de acuerdo a las pérdidas en porcentaje manifestado por los productores de plátano, en base a esto se logró obtener las pérdidas económicas por el bajo rendimiento del cultivo de plátano. Tal es así que, los productores que manifestaron sus pérdidas en un 5\% sufrieron pérdidas económicas por el bajo rendimiento desde S/. 310.33 a 1029.37 soles como es el caso de la comunidad nativa Santa Elisa y el caserío Santa Elena. Los productores que manifestaron sus pérdidas en un 90\% sufrieron pérdidas económicas de hasta S/.18 528.75 soles como es el caso del caserío Santa Elena.

\section{LISTA DE REFERENCIAS}

Ahrens, D. C. (2003). Meteorology today: an introduction to weather, climate, and the environment. 7. Australia: Thomson.

Altieri, M., \& Nicholls, C. (2008). Los impactos de cambio climático sobre las comunidades campesinas y de agricultores tradicionales y sus respuestas adaptativas. 3, 7, 7-23. Agroecología.

Amador, J., \& Alfaro, E. (2009). Métodos de reducción de escala: aplicaciones al tiempo, clima, variabilidad climática y cambio climático. 39- 52. Revista Iberoamericana de Economía Ecológica.

Bourguignon, F. (2003). The growth elasticity of poverty reduction: explaining heterogeneity across countries and time periods. Inequality and Growth: Theory and policy implications. (T. S. Eicher, \& S. J. (, Edits.) Cambridge, MIT Press. 
Brooks, N., \& Adger, W. N. (2003). Country level risk measures of climate related natural disasters and implicationes for adaptation to climate change. Reino Unido: Tyndall Center.

CAN. (Mayo de 2008). El Cambio Climático no tiene Fronteras. Impacto del Cambio Climático en la Comunidad Andina. . Comunidad Andina.

CÁRDENAS, D. F. (2011). Estudio de la cadena del plátano. MINAG. 150 . Lima, Perú. CEPAL. (2010). La economía del cambio climático en América Latina y el Caribe. .

CEPAL. (2013). Impactos potenciales del cambio climático sobre los granos básico en Centroamérica . D. F., México: CEPAL/Consejo Agropecuario Centroamericano $(\mathrm{CAC}) /$ Sistema de la Integración Centroamericana (SICA).

CLIMATICO, I. P. (2007). Impactos del Cambio Climatico. 27 .

CNCC - Comisión Nacional de Cambio Climático. (2002). Estrategia Nacional de Cambio Climático. 8.

CONAM. (1999). Perú: Vulnerabilidad Frente al Cambio Climático. Aproximaciones a la experiencia con el fenómeno El Niño. . Perú.

Corporación Colombiana de Investigación Agropecuaria, C. (2006). Manejo sostenible del cultivo del plátano. . Recuperado el 5 de Mayo de 2014, de http://www.corpoica.org.co/sitioweb/Archivos/Publicaciones/Cultivodelplta no.pdf

Dell Melissal, F. J., \& Olken., A. (Junio de 2008). Climate Change and Economic Growth: Evidence from the last half century. Working Paper 14132. National Bureau of Economic Research,.

DIRECCIÓN DE DESARROLLO AGROPECUARIO Y PROMOCIÓN DE LA INVERSIÓN PRIVADA. (2016). Diagnóstico de la cadena de valor del cultivo de plátano.

DIRECCION REGIONAL DE SALUD UCAYALI. (Junio de 2009 ). Análisis Situacional de Salud de Ucayali 2008. Información para la Toma de Decisiones. Dirección Regional de Epidemiología Ucayali.

EIECCP, C. (2013). Impacto Económico del Cambio Climático en la Agricultura Peruana. Estudio del Impacto Económico del Cambio Climático en el Perú. Documento no publicado. . 
FAO. (2008). La seguridad Alimentaria Mundial: Los Desafíos del Cambio Climático y la Bioenergía.

Fernandez, J. (2014). El cambio climático y los efectos económicos y financieros en el largo plazo en Latinoamérica. Lima , Perú.

Field, B., \& Azqueta, D. (1998). Economía y Medio Ambiente, Mc Graw Hill. 579 . Bogotá, Colombia.

Fisch, C., Marengo, J., \& Nobre, C. (1998). Uma revisão geral sobre o clima da Amazônia. Acta Amazônica. 28(2). 101 - 126.

García, C. (1999). "El Cambio Climático: Estado de la Cuestión y Aspectos Científicos". DT 8/99. . UEM-CEES Ediciones. .

Ghini, R., Bettiol, W., \& Hamada, E. (2011). Diseases in tropical plantation crops as affected by climate change: current knowledge and perspectives. 60, 122-132. Plant Pathol.

Herrera, M., \& Colonia, L. (2011). Manejo integral del cultivo de plátano. Lima, Perú: Universidad Nacional Agraria La Molina.

IICA . (1993). Instituto para la poscosecha de productos perecederos. Facultad de Agricultura. Universidad de Idaho Moscow, 129 . Idaho.

IPCC. (2001). Climate Change 2001: Impacts, adaptation and vulnerability. Contribution of working group II to the third assessment report of the Intergovernmental Panel on Climate Change. 1005. US: Cambridge University Press.

IPCC. (2007). Cimate change 2007: Impacts, Adaptation and Vulnerability. Contribution of Working Group II (WG2) to the Fourth Assessment Report of the Intergovernmental Panel on Climate Change.

Jones, P., \& Thornton, P. (2003). The potential impacts of climate change on maize production in Africa and Latin America in 2055. (13), 1, 51-59. Global Environmental Change. doi:10.1016/S0959-3780(02)00090-0

Lagos, P., Silva, Y., \& Nickl, E. (2004). El Niño y la precipitación en los Andes del Perú.1.17.

Locatelli, B. (2014). Bosques y adaptación al cambio climático. Recuperado el 1 de mayo del 2014, de la exposición realizado en la Universidad Nacional de Ucayali. .

Macharé, J., \& Ortlieb, L. (1993). Registros del Fenómeno El Niño en el Perú. Bull. Inst fr. études andines. 33-52. 
Matute, V., A, \& Guillen, J. (1992). Manual de producción de plátano. Lima, Cortes, Honduras, Fundación Hondureña de Investigación Agrícola. 109 .

Mendelsohn, R., Nordhaus, W., \& Shaw, D. (1992). The impact of climate on agriculture: a Ricardian approach. USA: Yale University. New Haven.

MINAGRI (Ministerio de Agricultura y Riego) . (2014). El Banano Peruano "Producto Estrella de Exportación”. Tendencias de la Producción y el Comercio del Banano en el Mercado Internacional y Nacional. Lima.

MINAM. (2010). Portal de Cambio Climático. Recuperado el 29 de Setiembre de 2011, de http://cambioclimatico.minam.gob.pe/cambioclimatico/sobre-cambioclimatico/que-impactos-tiene/en-el-peru/

Ministerio de Agricultura de Perú. (2011). Estudio del mercado de la cadena de plátano. 101. Perú.

Ministerio de Agricultura y Gobierno Regional de Ucayali . (2003). Programa nacional de banano orgánico y Ministerio de Agricultura. Lima.

Ministerio de Agricultura y Gobierno Regional de Ucayali. (2009). Plan estratégico Regional del Sector Agrario de Ucayali 2008-2012. 41 . Perú.

Montealegre, J. E. (2004). Escalas de la Variabilidad Climática. Bogotá: Insituto de Hidrología, Meterología y Estudios Ambientales - IDEAM.

OEIA-DRAU. (2017). Obtenido de http://www.draucayali.gob.pe/direccion-deestadistica-agraria.html

Rodriguez, G. M., \& Barrigh, O. (1979). Manual sobre el cultivo del plátano en la costa norte de Honduras. 54 (7). Tegucigalpa, Honduras: SIATSA.

SENAMHI-UNU. (2017). Obtenido de https://www.senamhi.gob.pe/?p=estaciones

Shapiama, S. (2008). Evaluación De Sistemas Agroecológicos Desarrollados En La Localidad De Saramiriza. 122. Iquitos, Perú.

Torres, L. (2010). Análisis Económico del Cambio Climático en la Agricultura de la Región: Principales Productos Agroexportables. Piura, Perú. Obtenido de http://old.cies.org.pe/files/documents/investigaciones/medioambienteyrecursosnaturales/analisis_economico_del_cambio_climatico_en_la_a gric ultura_de_region_piura.pdf

UNESCO. (1968). Citado en Some Russian economists on Return to Schooling and Experience. ARCADIUS /CARA>/: Readings in the Economics of Education. 
Vergara, \& otros, y. (Abril de 2013). The Climate and Development Challenge for Latin America and the Caribbean: Options for Climate-Resilient, Low-Carbon Development. Banco Interamericano de Desarrollo (BID).

Vergara, K. (2011). Variabilidad Climática, Percepción Ambiental y Estrategias De Adaptación De La Comunidad Campesina De Conchucos. 202. Ancash, Perú. 